PROCEEDINGS OF THE

AMERICAN MATHEMATICAL SOCIETY

Volume 136, Number 11, November 2008, Pages 3825-3829

S 0002-9939(08)09575-0

Article electronically published on July 3, 2008

\title{
ON A THEOREM OF BRAUER AND WIELANDT
}

\author{
KOICHIRO HARADA
}

(Communicated by Jonathan I. Hall)

\begin{abstract}
A classical theorem of Brauer and Wielandt which states the result under the assumption $G=[G, G]$ is extended to all finite groups.
\end{abstract}

Let $G$ be a finite group and $C_{1}, C_{2}, \ldots, C_{s}$ be the conjugacy classes of $G$. For a subset $S$ of $G$, define

$$
\bar{S}=\sum_{s \in S} s \in \mathbb{C} G
$$

where $\mathbb{C} G$ is the group ring of $G$ over the complex number field $\mathbb{C}$. On page 37 of W. Feit [Characters of Finite Groups, Benjamin, New York, 1967]), one finds a result. Feit writes on it: "The following striking consequence of (6.9) 1 was discovered by Braue12 and Wielandt. However, it is a result that seems to be difficult to use."

Theorem (Brauer and Wielandt). $\bar{C}_{1} \bar{C}_{2} \cdots \bar{C}_{s}=c \bar{G}$ for some $c \in \mathbb{C}$ if and only if $G=[G, G]=G^{\prime}$.

Let me add a corollary to this theorem.

Corollary. If $G=G^{\prime}$, then $C_{1} C_{2} \cdots C_{s}=G$.

For the proof of it, just observe that the $c$ in the theorem cannot be equal to 0 and every element of $G$ has an expression in the set $C_{1} C_{2} \cdots C_{s}$ at least once. The converse of this corollary also holds (see Corollary 5 ) but it does not seem to follow immediately from the theorem of Brauer and Wielandt (see "Acknowledgements" for the referee's comment).

I do not intend to argue with Feit on the latter part of the quotation given above, but I will show that a more general result can be proved. That is:

Corollary 3. For every finite group $G$, there exists $c \in \mathbb{C}$ such that $\bar{C}_{1} \bar{C}_{2} \cdots \bar{C}_{s}=$ $c \overline{\alpha G^{\prime}}$.

Received by the editors October 1, 2007.

2000 Mathematics Subject Classification. Primary 20-XX.

1 A theorem of Burnside quoted later in this note.

2 Proc. International Congress 1954, Vol. 1, pp. 1-9. The name of Wielandt is mentioned only in a footnote.

(C)2008 American Mathematical Society Reverts to public domain 28 years from publication 
Here $x_{j} \in C_{j}$ and $\alpha=x_{1} x_{2} \cdots x_{s}$. First of all, the constant $c$ in the theorem of Brauer and Wielandt can easily be computed and is 3

$$
c=\frac{\left|C_{1}\right|\left|C_{2}\right| \cdots\left|C_{s}\right|}{|G|} .
$$

The reason for this is that the product $\bar{C}_{1} \bar{C}_{2} \cdots \bar{C}_{s}$ is a positive linear combination of elements of $G$ and the sum of its coefficients is $\left|C_{1}\right|\left|C_{2}\right| \cdots\left|C_{s}\right|$. If it is a constant multiple of $\bar{G}$, then the $c$ must be equal to the value given above.

With this $c$ in mind, we can rewrite the relation of Brauer and Wielandt as follows:

$$
\frac{\bar{C}_{1}}{\left|C_{1}\right|} \frac{\bar{C}_{2}}{\left|C_{2}\right|} \cdots \frac{\bar{C}_{s}}{\left|C_{s}\right|}=\frac{\bar{G}}{|G|} .
$$

Therefore, in this form, the constant $c$ is reduced to 1 . Moreover, if we define, for a subset $S$ of $G$, the normalized sum:

$$
\hat{S}=\frac{\bar{S}}{|S|},
$$

then the result of Brauer and Wielandt can be restated as

$$
\hat{C}_{1} \hat{C}_{2} \cdots \hat{C}_{s}=\hat{G} \Longleftrightarrow G=G^{\prime} .
$$

For an arbitrary finite group $G$, define:

$$
\begin{gathered}
\hat{\Omega}_{G}=\hat{C}_{1} \hat{C}_{2} \cdots \hat{C}_{s}, \\
\alpha=x_{1} x_{2} \cdots x_{s},
\end{gathered}
$$

where $x_{i}$ is an (arbitrary but fixed once chosen) representative from the conjugacy class $C_{i}$ for $i=1,2, \ldots, s$. Note that there are many choices for the element $\alpha$, but the coset $\alpha G^{\prime}$ is uniquely determined. We will show in Theorem 2 (the main result of this paper) that $\hat{\Omega}_{G}=\alpha \hat{G}^{\prime}$ for every finite group $G$. In the original notation of the result of Brauer and Wielandt, this implies that $\bar{C}_{1} \bar{C}_{2} \cdots \bar{C}_{s}$ is proportional to $\overline{\alpha G^{\prime}}$ for every finite group $G$ (Corollary 3 ).

Examples. (1). Suppose $G$ is abelian. Then every conjugacy class $C_{i}$ is a singleton. Therefore

$$
\hat{\Omega}_{G}=\prod_{x \in G} x .
$$

Rearranging the product by combining $\left\{x, x^{-1}\right\}$, we obtain

$$
\hat{\Omega}_{G}=\prod_{t \in \operatorname{Inv}(G)} t,
$$

where $\operatorname{Inv}(G)$ is the set of all involutions of $G$. As is easily seen in the case of a four group and by induction in the general cases, $\prod_{t \in \operatorname{Inv}(G)} t=1$ if the Sylow 2-subgroup of $G$ is not cyclic and $\prod_{t \in \operatorname{Inv}(G)} t=t_{0}$, where $t_{0}$ is the unique involution of $G$ if the Sylow 2-subgroup of $G$ is (nontrivial) cyclic.

(2). $\hat{\Omega}_{S_{4}}=\hat{A}_{4}, \hat{\Omega}_{S_{5}}=\widehat{S_{5} \backslash A_{5}}$ and $\hat{\Omega}_{S_{8}}=\hat{A}_{8}$. In general, $\hat{\Omega}_{S_{n}}=\hat{A_{n}}$ if the number of odd partitions of $n$ is even and $\hat{\Omega}_{S_{n}}=\widehat{S_{n} \backslash A_{n}}$ in the contrary cases. This is proved in the Appendix.

\footnotetext{
${ }^{3}$ Brauer's paper in footnote 2 actually has this constant, although Feit did not mention it in his book.
} 
Define $\operatorname{Irr}(G)=\left\{\chi_{1}=1, \chi_{2}, \ldots, \chi_{s}\right\}$, the set of all irreducible characters of $G$ over $\mathbb{C}$ and let us compute the quantity $\hat{\Omega}_{G}$ for an arbitrary finite group $G$. Note that the element $\hat{G}$ is an idempotent of the center $Z(R)$ of the group ring $R=\mathbb{C} G$. $\hat{\Omega}_{G}$ is an element in $Z(R)$ also. Recall that $\operatorname{dim}(Z(R))=s$ and there are exactly $s$ algebra homomorphisms from $Z(R)$ to $\mathbb{C}$, each corresponding to an irreducible character of $G$. Let us denote those homomorphisms by $\omega_{1}, \omega_{2}, \ldots, \omega_{s}$ with $\omega_{i}$ corresponding to the irreducible character $\chi_{i}$ of $G$ for $i=1,2, \ldots, s$. It is well known that

$$
\omega_{i}\left(\bar{C}_{j}\right)=\frac{\left|C_{j}\right| \chi_{i}\left(x_{j}\right)}{\chi_{i}(1)}
$$

and so

$$
\omega_{i}\left(\hat{C}_{j}\right)=\frac{\chi_{i}\left(x_{j}\right)}{\chi_{i}(1)}
$$

Let $\left\{e_{i} \mid i=1,2, \ldots, s\right\}$ be the set of all primitive idemponents of $Z(R)$. Then

$$
Z(R)=\bigoplus_{i=1}^{s} \mathbb{C} e_{i}
$$

Moreover, we have

$$
\omega_{i}\left(e_{j}\right)=\delta_{i j}
$$

where $\delta_{i j}$ is the Kronecker delta.

Write $\hat{\Omega}_{G}$ using a basis $\left\{e_{1}, e_{2}, \ldots, e_{s}\right\}$ of $Z(R)$ :

$$
\hat{\Omega}_{G}=\sum_{i=1}^{s} a_{i} e_{i}, a_{i} \in \mathbb{C} .
$$

Applying $\omega_{i}$ to both sides of the equality above, we obtain

$$
\omega_{i}\left(\hat{\Omega}_{G}\right)=\prod_{j=1}^{s} \frac{\chi_{i}\left(x_{j}\right)}{\chi_{i}(1)}=a_{i} .
$$

A theorem of Burnside states that if $\operatorname{deg}\left(\chi_{i}\right) \geq 2$, then there exists $j$ such that $\chi_{i}\left(x_{j}\right)=0$. Therefore $\omega_{i}\left(\hat{\Omega}_{G}\right)=a_{i}=0$ in this case. On the other hand, if $\operatorname{deg}\left(\chi_{i}\right)=1$, then

$$
a_{i}=\chi_{i}\left(\prod_{j=1}^{s} x_{j}\right)=\chi_{i}(\alpha) .
$$

This implies $a_{i} \neq 0$ if and only if $\operatorname{deg}\left(\chi_{i}\right)=1$. Rearrange the indices $i$ so that $\left\{\chi_{1}=1, \chi_{2}, \ldots, \chi_{r}\right\}$ is the set of all irreducible characters of degree 1 .

Theorem 1. If $\alpha \in G^{\prime}$, then $\hat{\Omega}_{G}$ is the sum of the primitive idempotents of $Z(R)$ corresponding to the irreducible characters of degree 1 :

$$
\hat{\Omega}_{G}=\sum_{i=1}^{r} e_{i} .
$$

Proof. If $\alpha \in G^{\prime}$, then all coefficients $a_{i}$, for $i=1,2, \ldots, r$, are equal to 1 . Hence the result follows. 
The formula for the idempotent $e_{i}$ is well known and given by

$$
e_{i}=\frac{\chi_{i}(1)}{|G|} \sum_{x \in G} \chi_{i}\left(x^{-1}\right) x
$$

If $\chi_{i}$ is taken to be the trivial character $\chi_{1}=1$, then $a_{1}=1$ and

$$
e_{1}=\frac{1}{|G|} \sum_{x \in G} x=\hat{G} \text {. }
$$

Therefore,

$$
\hat{\Omega}_{G}=\hat{G}+\sum_{i=2}^{r} a_{i} e_{i} .
$$

The result of Brauer and Wielandt can now be obtained easily. If $G=G^{\prime}$, then $G$ possesses no nontrivial characters of degree 1 and so $\hat{\Omega}_{G}=\hat{G}$. On the other hand, if $G$ possesses a nontrivial character $\chi_{i}$ of degree 1 , then as shown above $a_{i} \neq 0$ and so $\hat{\Omega}_{G} \neq \hat{G}$. This proves the result of Brauer and Wielandt.

Let us study $\hat{\Omega}_{G}$ a little more. We have the following result.

Theorem 2. $\hat{\Omega}_{G}=\alpha \hat{G}^{\prime}$.

Proof. As stated above, we have in general

$$
\hat{\Omega}_{G}=\sum_{i=1}^{r} a_{i} e_{i}, a_{i}=\chi_{i}(\alpha) .
$$

Moreover,

$$
e_{i}=\frac{1}{|G|} \sum_{x \in G} \chi_{i}\left(x^{-1}\right) x
$$

Therefore,

$$
\hat{\Omega}_{G}=\frac{1}{|G|} \sum_{i=1}^{r}\left(\sum_{x \in G} \chi_{i}\left(\alpha x^{-1}\right)\right) x .
$$

Note again that $\chi_{i}$ is of degree 1 for $i=1,2, \ldots, r$ and so $\chi_{i}(\alpha) \chi_{i}\left(x^{-1}\right)=\chi_{i}\left(\alpha x^{-1}\right)$. Replacing $\alpha x^{-1}$ by $y^{-1}$, this can be rewritten as

$$
\hat{\Omega}_{G}=\frac{1}{|G|} \sum_{y \in G}\left(\sum_{i=1}^{r} \chi_{i}\left(y^{-1}\right)\right) y \alpha .
$$

Note that the characters of $G$ of degree 1 can be identified with the characters of the abelian group $G / G^{\prime}$. Therefore, the orthogonality relations of characters of $G / G^{\prime}$ implies: if $y \notin G^{\prime}$, then $\sum_{i=1}^{r} \chi_{i}\left(y^{-1}\right)=0$ and $\sum_{i=1}^{r} \chi_{i}\left(y^{-1}\right)=\left[G: G^{\prime}\right]$ if $y \in G^{\prime}$. We now conclude

$$
\hat{\Omega}_{G}=\frac{1}{|G|} \sum_{y \in G^{\prime}}\left[G: G^{\prime}\right] y \alpha=\frac{1}{|G|}\left[G: G^{\prime}\right]\left(\sum_{y \in G^{\prime}} y\right) \alpha=\alpha \hat{G}^{\prime} .
$$

This completes the proof of Theorem 2 .

Theorem 2 (the following corollary also) is a generalization of the result of Brauer and Wielandt. In fact, if $G=G^{\prime}$, then $\alpha \in G^{\prime}$ and so $\hat{\Omega}_{G}=\alpha \hat{G}^{\prime}=\hat{G}$. On the other hand, if $\hat{\Omega}_{G}=\hat{G}$, then $\alpha \hat{G}^{\prime}=\hat{G}$. Hence $\alpha G^{\prime}=G$ as sets. This implies $G=G^{\prime}$.

Corollary 3. For every finite group $G$, there exists $c \in \mathbb{C}$ such that $\bar{C}_{1} \bar{C}_{2} \cdots \bar{C}_{s}=$ $c \overline{\alpha G^{\prime}}$. $\left(c=\left|C_{1}\right|\left|C_{2}\right| \cdots\left|C_{s}\right| /\left|G^{\prime}\right|.\right)$

Proof. This is a trivial consequence of Theorem 2 since $\alpha \bar{G}^{\prime}=\overline{\alpha G^{\prime}}$. 
Corollary 4. For every finite group $G$, it follows that $C_{1} C_{2} \cdots C_{s}=\alpha G^{\prime}$. In particular, if $\alpha \in G^{\prime}$, then $C_{1} C_{2} \cdots C_{s}=G^{\prime}$.

Proof. Considering subsets of $G$ in lieu of sums of group elements in $\mathbb{C} G$, we see that this is an easy consequence of Corollary 3.

We can now prove the converse of the corollary to the result of Brauer and Wielandt.

Corollary 5. If $C_{1} C_{2} \cdots C_{s}=G$, then $G=G^{\prime}$.

Proof. Noting that $\alpha G^{\prime}$ is a single coset of $G^{\prime}$, we see that $\alpha G^{\prime}=G$ implies $G=$ $G^{\prime}$.

Results such as these last two corollaries will not be easy to prove by a pure group-theoretical argument.

Lemma 6. $\alpha^{2} \in G^{\prime}$.

Proof. By definition $\alpha=x_{1} x_{2} \cdots x_{s}$. Write $\bar{G}=G / G^{\prime}$ (by abuse of notation). So $\bar{\alpha}=\bar{x}_{1} \bar{x}_{2} \cdots \bar{x}_{s}$. Note that the coset $\bar{x}_{j}=x_{j} G^{\prime}$ is a union of conjugacy classes of $G$. If $\bar{x}_{j} \neq \bar{x}_{j}^{-1}$, then the cosets $x_{j} G^{\prime}$ and $x_{j}^{-1} G^{\prime}$ are disjoint. Therefore, the corresponding products in $\bar{\alpha}$ reduce to $\overline{1}$, and so in the expression of $\bar{\alpha}$ only involutions of $G / G^{\prime}$ can actually appear nontrivially. Hence the lemma follows.

Corollary 7. If $G / G^{\prime}$ is of odd order, then $\alpha \in G^{\prime}$ and $\hat{\Omega}_{G}=\hat{G}^{\prime}$.

Proof. Since $\alpha^{2} \in G^{\prime}$ by Lemma 6 , we have $\alpha \in G^{\prime}$ under the condition of this lemma. The corollary now follows from Theorem 2 .

Corollary 8. The order of $G$ is odd if and only if $\hat{\Omega}_{H}=\hat{H}^{\prime}$ for every subgroup $H$ of $G$.

Proof. If $|G|$ is odd, the assertion follows from Corollary 7. If $|G|$ is even, then $G$ contains a subgroup $H$ of order 2. It is trivial that $\hat{\Omega}_{H}=h \neq \hat{H}^{\prime}$ where $h$ is the unique involution of $H$. This proves the corollary.

Appendix. Earlier we stated $\hat{\Omega}_{S_{n}}=\hat{A}_{n}$ if there is an even number of odd partitions of $n$ and $\hat{\Omega}_{S_{n}}=\widehat{S_{n} \backslash A_{n}}$ in the contrary cases. To prove this result, we only need to determine whether $\alpha \in A_{n}$. Hence the result.

\section{ACKNOWLEDGEMENTS}

M. Kiyota and M. Miyamoto made a valuable suggestion which led to an improvement of this paper. The referee noted that Corollary 5 has an easy grouptheoretic proof. It goes as follows. If $g_{i} \in C_{i}$, then $g_{i} G^{\prime}=x_{i} G^{\prime}$. It follows that $C_{1} C_{2} \cdots C_{s} \subseteq \alpha G^{\prime}$. So if $C_{1} C_{2} \cdots C_{s}=G$, then $G$ is contained in a single coset of $G^{\prime}$. Thus $G=G^{\prime}$. We thank the referee.

Department of Mathematics, Ohio State University, Columbus, Ohio 43210

Current address: 2-2-14 Toshinden, Suruga-ku, Shizuoka-shi, 421-0112 Japan

E-mail address: haradako@math.ohio-state.edu 